\title{
MULTI-LEVEL PLURALS AND DISTRIBUTIVITY
}

\author{
Remko Scha and David Stallard \\ BBN Laboratories Inc. \\ 10 Moulton St. \\ Cambridge, MA 02238 \\ U.S.A.
}

\begin{abstract}
We present a computational treatment of the semantics of plural Noun Phrases which extends an earlier approach presented by Scha [7] to be able to deal with multiple-level plurals ("the boys and the giris". "the juries and the committees". etc. $)^{1} W_{\theta}$ argue that the arbitrary depth to which such plural structures can be nested creates a correspondingly arbitrary ambiguity in the possibilities for the distribution of verbs over such NPs. We present a recursive transiation rule scheme which accounts for this ambiguity, and in particular show how it allows for the option of "partial distributivity" that collective verbs have when applied to such plural Noun Phrases.
\end{abstract}

\section{INTRODUCTION}

Syntactically parallel utterances which contain plural noun phrases often require entirely different semantic treatments depending upon the particular verbs (or adjectives or prepositions) that these plural NPs are combined with. For example, while the sentence "The boys walk" would have truth-conditions $\theta x-$ pressed by: 2

\section{$\forall x \in$ BOYS: WALK $[x]$}

the very similar sentence "The boys gather" could not be translated this way. Its truth-conditions would instead have to be expressed by something like:

\section{GATHER[BOYS]}

since only a group can "gather", not one person by himself.

It is common to call a verb such as "walk" a "distributive" verb. while a verb such as "gather" (or "disperse" or intransitive "meet") is called a

\footnotetext{
'The work presented here was supported under DARPA contracts *N00014-85-C-0016 and *N00014-B7-C-0085. The views and conclusions contained in this document are those of the authors and should not be interpreted as necessarily representing the official policies, either expressed or implied, of the Detense Advanced Research Projects Agency or the United States Government.
}

${ }^{2}$ We ignore here the discourse issues that bear on the interpretation of definite NPs "collective" verb. The collective/distributive distinction raises an important issue: how to treat the semantics of plural NPs uniformly.

An earlier paper by Scha ("Distributive, Collective and Cumulative Quantification" [7], hereinafter "DCC") presented a formal treatment of this issue which exploits an idea about the semantics of plural NP's which is due to Bartsch [1]: plural NP's are always interpreted as quantifying over sets rather than individuals; verbs are correspondingly always treated as collective predicates applying to sets. Distributive verbs are provided with meaning postulates which relate such collective applications to applications on the constituent individuals.

The present paper describes an improved and extended version of this approach. Two important problems are addressed. First, there is the problem of ambiguity: the need to allow for more than one distribution pattern for the same verb. Second, there is the problem of "multi-level plurality": the consequences which arise for the distributive/collective distinction when one considers conjoined plural NPs such as "The boys and the girls".

Both issues are addressed by a two-level system of semantic interpretation where the first level deals with the semantic consequences of syntactic structure and the second with the lexically specific details of distribution.

The treatment of plural NPs described in this paper has been implemented in the Spoken Language System which is being developed at BBN. The system provides a natural language interiace to a database/graphic display system which is used to access information about the capabilities and readiness conditions of the ships in the Pacific Feet of the US Navy.

The remainder of the paper is organized as follows:

Section 2 discusses previous methods of handling the distributive/collective distinction, and shows their limitations in dealing with the problems mentioned above.

Section 3 presents our two-level semantics approach, and shows how it handles the problem of ambiguity. 
Section 4 shows how a further addition to the twolevel system - recursive enumeration of lexical meanings - handles the multi-level plural problem.

Section 5 presents the algorithm that is used and Section 6 presents conclusions.

\section{BACKGROUND}

\subsection{An Approach to Distributivity}

One possible way to generate the correct readings for "The boys walk" vs. "The boys gather" is due to Bennett [2]. Verbs are sub-categorized as either collective or distributive. Noun phrases consisting of "the" + plural then have two readings; a "set" reading if they are combined with a collective verb and a universal quantification reading if they are combined with a distributive verb.

Scha's "Distributive, Collective, and Cumulative Quantification" ("DCC") showed that this approach, while plausible for intransitive verbs, breaks down for the two-argument case of transitive verbs [7]. Consider the example below:

"The squares contain the circles"

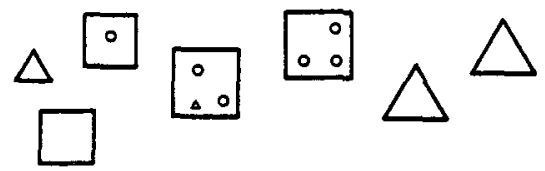

This sentence has a reading which can be approximately paraphrased as "Every circle is contained in some square" so that in the world depicted above the sentence would be considered true.

The truth-conditions which Bennett's approach would predict, however, are expressed by the formula:

$\forall x \in$ SQUARES: $\forall y \in$ CIRCLES: CONTAIN $[x, y]$

which obviously does not correspond to the state of affairs pictured above.

"DCC" avoids this problem by not generating a distributive translation directly. Noun phrases, regardless of number, quantify over sets of individuals: a singular noun phrase simply quantifies over a singleton set. Nouns by themselves denote sets of such singleton sets. Thus, both "square" and "squares" are translated as:

\section{SQUARES *}

in which the asterisk operator "*N" creates the set of singleton subsets of "SQUARES".

Verbs can now be uniformly typed to accept sets of individuals as their arguments. The collective/distributive distinction consists solely in whether a verb is applied to a large set or to a singleton set.
Determiner translations are either distributive or collective depending upon whether they apply the predicate to the constituent singletons or to their union. Some determiners are unambiguously distributive, for example the translation for "each":

$$
(\lambda X:(\lambda P: \forall x \in X: P(x)))
$$

Other determiners - "all", "some" and "three" - are ambiguous between transiations which are distributive and translations which are collective. Plural "the", on the other hand, is unambiguously collective, and has the translation:

$$
\text { ( } \lambda X:(\lambda P: P(U(X))))
$$

where " $U$ " takes a set of sets and delivers the set which is their union.

The following is a list of sentences paired with their translations under this scheme:

$$
\begin{aligned}
& \text { The boys walk } \\
& \text { WALK(BOYS) } \\
& \text { Each boy walks } \\
& \forall x \in \text { BOYS*: WALK( } x \text { ) } \\
& \text { The boys gather } \\
& \text { GATHER(BOYS) }
\end{aligned}
$$

The squares contain the circles CONTAIN(SOUARES.CIRCLES)

For "the" + plural NP's we thus obtain analyses which are. though not incorrect. perhaps more vague than one would desire. These analyses can be further spelled out by providing distributive predicates, such as "WALK" and "CONTAIN", with meaning postulates which control how that predicate is distributed over the constituents of its argument. For example, the meaning postulate associated with "WALK" could be:

$$
\text { WALK }[x]=[\because(x)>0] \wedge[\forall y \in x: \text { WALK}[y]
$$

which, when applied to the above translation "WALK[BOYS]", gives the result:

$$
\text { [\#(BOYS) > 0]^[ } \forall y \in \text { BOYS*: WALK[y]] }
$$

which represents the desired distributive truthconditions.

The meaning postulate for "CONTAIN" could be:

$$
\text { CONTAIN }[u, v]=\forall y \in v: \exists x \in u^{*}: \text { CONTAIN }[x, y]
$$

This meaning postulate may be thought of as expressing a basic fact about the notion of containment; namely that one composite object is "contained" by another if every every part of the first is contained in some part of the second. Application of this meaning postulate to the translation

\section{CONTAIN[SOUARES,CIRCLES]}

gives the final result:

$$
\forall y \in \text { SOUARES }: ~ \exists x \in \text { CIRCLES }: \text { CONTAIN }[x, y]
$$

which expresses the truth-conditions we originally 
desired; namely those paraphrasable by "Every circle is contained by some square".

In general, it is expected that different verbs will have different meaning postulates, corresponding to the different facts and beliefs about the world that pertain to them.

\subsection{Problems}

\section{Conjunctive Noun Phrases}

"DCC" only treated plural Noun Phrases (such as "the boys" and "some gir's"), but did not deal with conjunctive Noun Phrases ("John, Peter and Bill", "the boys and the girls", or "the committees and the juries"). It is not immediately clear how a treatment of them would be added. Note that a PTQ-style ${ }^{3}$ treatment of the NP "John and Peter":

\section{$\lambda P$ : $P$ (John') ^ $P\left(\right.$ Peter' $\left.^{\prime}\right)$}

would encounter serious difficulties with a sentence like "John and Peter carried a piano upstairs". Here it would predict only the distributed reading, yet a collective reading is the desired one.

It would be more in the spirit of the treatment in "DCC" to combine the denotations of the NPs that are conjoined by some form of union. For example. "John and Peter". "The boys and the girls" might be translated as:

$$
\begin{aligned}
& \lambda P: P(\text { John' ,Peter' }\}) \\
& \lambda P \text {. P(BOYS U GIRLS })
\end{aligned}
$$

For a sentence like "The boys and the girts gather" this prevents what we call the "partially" distributive" reading - namely the reading in which the boys gather in one place and the giris in another.

For this reason, it seems incorrect to assimilate all NP denotations to the type of sets of individuals. Noun phrases like "The boys and the girls" or "The juries and the committees", are what we call "multilevel plurals": they have internal structure which cannot be abolished by assimilation to a single set.

Note that the plural NP "the committees" is a multi-level plural as well, even though it is not a conjunction. The sentence "The committees gather" has a partially distributive reading (each committe gathers separately) analogous with the partially distributive reading for "The boys and girls gather" above.

\section{Ambiguity and Discourse Effects}

The final problem for the treatment in "DCC" has to do with the meaning postulates themselves. These always dictate the same distribution pattern for any

\footnotetext{
"We use the word "style" because Montague's original paper [6] only conjoined term phrases with "or". The extension to "and". however, is straightonwand.
}

verb. yet it does not seem plausible that one could finally decide what this should be. since the beliefs and knowledge about the world from which they are derived are subject to variation from speaker to speaker.

Variability in distribution might also be imposed by context. Consider the sentence "The children ate the pizzas" and a world depicted by the figure in 2.1 where the squares represent children, and the circles, pizzas. Now there will be different quantificational readings of the sertence. The question "What did the children eat?" might be reasonably answered by "The pizzas". If one were to instead ask "Who ate the pizzas?" (with a view, perhaps, to establishing individual guilt) the answer "The children" would not be as felicitous, since the picture includes one square (child) not containing anything.

It is to these problems with meaning postulates that we now turn in Section 3. The solution presented there is then used in Section 4, where we present our solution to the NP-conjunction/multi-level plural problem.

\section{THE AMBIGUITY PROBLEM}

\subsection{The Problem with Meaning Postulates}

That certain predicates may have different distributive expansions in different contexts cannot be captured by meaning postulates: since meaning postulates are stipulated to be true in all modeis it is logically incoherent to have several, mutually incompatible meaning postulates for the same constant. ${ }^{4}$

An alternative might be to retreat from the notion of meaning postulates per se, and view them instead as some form of conventional implicatures which are "usually" or "often" true. While it is impossible to have alternative meaning postulates, it is easier to imagine having alternative implicatures.

For a semantics which aspires to state specific truth-conditions this is not a very attractive position. We prefer to view these as altemative readings of the sentence, stemming from an open-ended ambiguity of the lexical items in question - an ambiguity which has to do with the specific details of distributions.

Since this ambiguity is not one of syntactic type it does not make sense (in either explanatory or computational terms) to multiply lexical entries on its behalf. Rather, one wants a level of representation in which these distributional issues are left open, to be resolved by a later stage of processing.

\footnotetext{
${ }^{4}$ One might try to combine them into a singie meaning postulate by logical disjunction. We have indicated before [9] why this approach is not satisfactory.
} 


\subsection{Two Levels of Semantic Interpretation}

To accommodate this our system employs two stages of semantic interpretation, using a technique for coping with lexical ambiguity which was originally developed for the Question-Answering System PHLIOA [3] [8]. The first stage uses a context-free grammar with associated semantic rules to produce an expression of the logical language EFL (for English-Oriented Formal Language). EFL includes a descriptive constant for each word in the lexicon, however many senses that word may have. Hence EFL is an ambiguous logical language; in technical terms this means either that the language has a model-theory that assigns multiple denotations to a single expression [5], or that its expressions are viewed as schemata which abbreviate sets of possible instance-expressions. [9]

The second stage translates the EFL expression into one or more expressions of WML (for World Model Language). WML, while differing syntactically from EFL only in its descriptive constants, is unambiguous. and inciudes a descriptive constant for each primitive concept of the application domain in question. A set of translation rules relates each ambiguous constant of EFL to a set of WML expressions representing its possible meanings. Translation of EF expressions to WML expressions is effected by producing all possible combinations of constant substitutions and removing those which are "semantically anomalous", in a sense which we will shortly define.

EFL and WML are instantiations of a higher-order logic with a recursive type system. In particular, if $\alpha$ and $\beta$ are types, then:

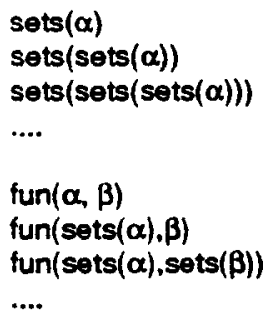

are all types. The type "sets $(\alpha)^{n}$ is the type of sets whose elements are of type $\alpha$. The type "FUN $(\alpha, \beta)$ " is the type of functions from type $\alpha$ to type $\beta$.

Every expression has a type. which is computed from the type of its sub-expressions. Types have domains which are sets; whatever denotation an expression can take on must be an element of the domain of its type. Some expressions, being constructed from combinations of sub-expressions of inappropriate types, are not meaningful and are said to be "semantically anomalous". These are assigned a special type, called NULL-SET, whose domain is the empty set.

For example, if " $F$ " is an expression of type fun $(\alpha, \beta)$ and "a" is an expression of type $\chi$ whose domain is disjoint from the domain of $\alpha$, then the ex- pression " $F(a)$ " representing the application of "F" to " $\mathrm{a}$ " is anomalous and has the type NULL-SET.

For more details on these formal languages and their associated type system, see the paper by Landsbergen and Scha [5].

\subsection{Translation Rules Instead of Meaning Postulates}

We are now in a position to replace the meaning postulates of the "DCC" system with their equivalent EFL to WML translation rules. For example, the original treatment of "contain" would now be represented by the translation rule:

CONTAIN $\Rightarrow \lambda u, v . \forall y \in v^{*}: \exists x \in u^{*}:$ CONTAIN $^{\prime}[x, y]$

Note that the constant "CONTAIN" " on the right-hand side is a constant of WML. and is notationally separated from its EFL counterpart by a prime-mark.

The device of translation rules can now be brought to bear on the problem mentioned in section 2.2. namely the distributional ambiguity (in context) of the transitive vert "eat". The reading which allows an exception in the first argument would be generated by the translation rule:

$$
\text { EAT } \Rightarrow \lambda u, v: \forall y \in v^{*}: \exists x \in u^{*}: \text { EAT' }[x, y]
$$

while the reading which allows no such exception would be:

$$
\begin{aligned}
& \text { EAT } \Rightarrow \\
& \lambda u, v:\left[\forall x \in v: \exists y \in u^{*}: \text { EAT' }[y, x]\right] \wedge \\
& {\left[\forall x \in u^{*}: \exists y \in v^{*}: \text { EAT' }[x, y]\right]}
\end{aligned}
$$

We call this a "leave none out" translation. When applied to the sentence "The children ate the pizzas" this generates the reading where all children are guilty.

By using this device of translation rules a verb may be provided with any desired (finite) number of alternative distribution patterns.

The next section. which presents this paper's treatment of the multiple plurals problem, will make use of a slight modification of the foregoing in which the translation rules are allowed to contain EFL constants on their right-hand sides as well as their left, thus making the process recursive.

\section{MULTIPLE LEVELS OF PLURALITY}

\subsection{Overview}

As we have seen in Section 2.2. Utterances which contain multi-level plurals sometimes give rise to mixed collective/distributive readings which cannot be accounted for without retaining the separate semantic identity of the constituents. 
Consider, for instance, the sentence "The juries and the committees gather". This has three readings: one in which each of the juries gathers alone and each of the committees gathers alone as well (distribution over two levels), another in which all persons who are committee members gather in one place and all persons who are jurors gather in another place (distribution over one level), and finally a third in which all jurors and committee members unite in one large convention (completely collective). It seems inescapable, therefore, that the internal multi-level structure of NPs has to be preserved.

Indeed. it can be argued that the number of levels necessary is not two or three but arbitrary. As Landman [4] has pointed out. conjunctions can be arbitrarily nested (consider all the groupings that are possible in the NP "Bob and Carol and Ted and Alice"!). Therefore, the sets which represent collective entities must, in principle, be allowed to be of arbitrary complexity. This is the view we adopt.

Allowing arbitrary complexity in the structure of collective entities creates a problem for specifying the distributive interpretations of collective predicates: they can no longer be enumerated by finite lists of translation nules. An arbitrary number of levels of structure means an arbitrary number of ways to distribute, and these cannot be finitely enumerated.

In order to handle these issues it is necessary to extend the ambiguity treatment of the previous subsection so that, as is advocated in [9], it recursively enumerates this infinite set of alternatives. In order to do this we must allow EFL constants to also appear on the right-hand side of translation rules as well as on the left.

In the next sub-section we present such a recursive EFL constant. Its role in the system is to deal with distributions over arbitrarily complex plural structures.

\subsection{The PARTS Function}

For any complex structure there is generally more than one way to decompose it into parts. For example, the structure

\{ John,Peter,Bill\},\{Mary,Jane,Lucy\}\}

can be viewed as either having two parts - the sets '(John,Peter.Bill\}' and '(Mary,Jane,Lucy\}' - or six - the six people John,Peter,Bill,Mary,Jane, and Lucy.

These multiple perspectives on a complex entity are accommodated in our system by the EFL function PARTS. This function takes a term, simple or complex, and returns the set of "parts" (that is, mathematical "parts") making it up. Because there is in general more than one way to decompose a composite entitity into parts, this is an ambiguous term which can be expanded in more than one way. In addition, because the set-theoretic structures corresponding to plural en- tities can be arbitrarily complex, some expansions must be recursive, containing PARTS itself on the right-hand side.

The expansions of PARTS are:

1. PARTS $[x]=>x \quad$ (where $x$ an individual)

2. PARTS $[s]=>$ (for: $s$, collect: PARTS)

(where s a set)

3. PARTS $[s] \Rightarrow$ U(for: $s$, collect: PARTS)

(where s a set)

\section{PARTS $[x] \Rightarrow F[x]$}

Rule (1) asserts that any atomic entity is indivisible, that is, is its own sole part (remember, we are talking about mathematical, not physical parts here). Rules (2) and (3) range over sets and collect together the set of values of PARTS for each member; rule (3) differs in that it merges these into a single set with the operator ' $U$ '. ' $U$ ' takes a set of sets and returns their union. In rule (4) " $F$ " is a descriptive function. This rule is included to handle notions like membership of a committee, etc.

Suppose PARTS is applied to the structure:

\{ John,Peter,Bill\}, \{Mary,Jane,Lucy\} \}

corresponding, perhaps, to the denotation of the NP The boys and the girls". The alternative sets of parts of this structure are:

\section{(1) \{John,Peter,Bill,Mary,Jane,Lucy\} \\ (2) \{ \{John,Peter,Bill\}, \{Mary,Jane,Lucy\} \}}

Let us see how these are produced by recursively expanding the function PARTS. Suppose we invoke rule (3) to begin with. This produces:

U(for: \{ \{John, Peter,Bill\}, \{Mary,Jane,Lucy\} \}, collect: PARTS)

Now suppose we invoke rule (2) on this, resulting in:

U(for: $\{$ \{John,Peter,Bill\},\{Mary,Jane,Lucy\}\}, collect: $\lambda x$. (for: $x$, collect: PARTS))

In the final step, we invoke rule (1) to produce:

U(for:\{ \{John,Peter,Bill\},\{Mary,Jane,Lucy\}\} collect: $\lambda x$. (for: $x$. collect: $\lambda x: x$

This expression simplifies to:

\{John,Peter,Bill,Mary,Jane,Lucy\}

which is just the expansion (1) above.

Now suppose we had invoked rule (2) to start with, instead of rule (3). This would produce the expansion:

for: \{ \{John,Peter,Bill\},\{Mary,Jane,Lucy\}\}, collect: PARTS

The rest of the derivation is the same as in the first 
example. We invoke rule (2) to produce the expansion

for: \{ \{John,Peter,Bill\}.\{Mary,Jane,Lucy\} \},

collect: $\lambda x$. (for: $x$, collect: PARTS)

Rule (1) is then invoked:

for: ( \{John.Peter.Bill\}, (Mary,Jane,Lucy\} \}, collect: $\lambda x$ : (for: $x$, collect: $\lambda x, x)$

There are now no more occurrences of PARTS left. This expression reduces by logical equivalence to:

\{ \{John.Peter,Bill, \{Mary,Jane,Lucy\} \}

which is just the expansion (2).

We now proceed to the distributing translation rules for verbs, which make use of the PARTS function in order to account for the multiple distributional readings economically.

\subsection{The Distributing Translation Rules}

The form below is an example of the new scheme for the translation rules, a translation which can cope with the problem originally posed in section 2.1, "The squares contain the circles":

\section{CONTAIN $\rightarrow$}

$\lambda u, v: \forall x \in$ PARTS[\{v\}]:

$\exists y \in$ PARTS $\{$ u $\}]:$ CONTAIN' $[y, x]$

This revised system can now cope with multi-level plural arguments to the verb "contain". Suppose we are given The squares contain the circles and triangles". The initial translation is then:

$$
\begin{gathered}
\forall x \in \text { PARTS }\{\{\text { CIRCLES,TRIANGLES }\}\}]: \\
\exists y \in \text { PARTS[\{SQUARES }\}]: \\
\text { CONTAIN' }[y, x]
\end{gathered}
$$

The ranges of the quantifiers each contain an occurrence of the PARTS function, so it is ambiguous as to what they quantify over. Note, however, that the WML predicate CONTAIN' is typed as being applicable to individuals only. Inappropriate expansions for the quantifier ranges therefore result in anomalous expressions which the translation algorithm filters out.

The first range restriction:

$$
\text { PARTS[\{CIRCLES,TRIANGLES\}\}] }
$$

is expanded to:

$$
\begin{aligned}
& \text { U(for: }\{\{C I R C L E S . T R I A N G L E S\}\}, \\
& \text { collect: } \lambda x \text { U(for: } x \text {, } \\
& \text { collect: } \lambda x \text { (for: } x \text {, collect: } \lambda x . x)) \text { ) }
\end{aligned}
$$

by a sequence of expansion rule applications

(3), (3), (2), (2), and (1). This final form is equivalent to:

\footnotetext{
${ }^{5}$ Note one other modification with respect to the treatment presented in section 2.1: predicates translating verbs are now atlowed to operate on individuals insteed of sets oniy
}

\section{U(CIRCLES,TRIANGLES)}

The other restriction. 'PARTS[[SQUARES]]', is reduced by similar means to just 'SQUARES'. We have, finally:

$$
\begin{aligned}
& \forall x \in \text { U(CIRCLES,TRIANGLES): } \\
& \exists y \in \text { SQUARES: CONTAIN' }[y, x]
\end{aligned}
$$

which expresses the desired truth-conditions.

\subsection{Partial Distribution of Collective Verbs}

Let us take up again the example The juries and committees gather". Recall that this has three readings: one in which each deliberative body gathers apart. another in which the various jurors combine in a gathering and the various committee members combine separately in another gather, and finally, one in which all persons concerned. be they jurors or committee members, come together to form a single gathering.

These readings are accounted for by the following translation rule for GATHER:

$$
\text { GATHER } \Rightarrow \lambda x . \forall y \in \text { PARTS }[\{x\}]: \text { GATHER }[y]
$$

Applying this rule to the initial translation:

$$
\text { GATHER[\{\{JURIES,COMMITTEES\}\}] }
$$

produces the expression:

$$
\begin{aligned}
& \forall y \in \text { PARTS }[\{[J U R I E S, C O M M I T T E E S]\}]: \\
& \text { GATHER' }[Y]
\end{aligned}
$$

The various readings of this now depend upon what the range of quantification is expanded to. This must be a set of sets of persons in order to fit the type of GATHER', which is a predicate on sets of persons.

We will now show how the PARTS function derives the decompositions that allow each of these readings. Because of the collective nature of the terms "jury" and "committee" we will use rule (4), which uses an arbitrary descriptive function to decompose an element.

Suppose that 'JURIES' has the extension ' $\left[i_{1}, j_{2}, j_{3}\right\}$ ' and 'COMMITTEES' has the extension ' $\left\{c_{1}, c_{2}, c_{3}\right\}$ '. Suppose also that the the descriptive function 'MEMBERS-OF is available, taking an organization such as a jury or committee onto the set of people who are its members. Let it have an extension corresponding to:

$$
\begin{aligned}
& j_{1} \rightarrow\{a, b, c\} \\
& j_{2} \rightarrow\{d, e, f\} \\
& j_{3} \rightarrow\{g, h, i\} \\
& c_{1} \rightarrow\{j, k, l\} \\
& c_{2} \rightarrow\{m, n, o\} \\
& c_{3} \rightarrow\{p, q, r\}
\end{aligned}
$$

where the letters a,b,c, etc. represent persons.

The derivation (3).(3),(2).(4) yields the first of the readings above, in which the verb is partially dis- 
tributed over two levels. The range of quantification has the extension:

$$
\{\{a, b, c\},\{d, e . f\},\{g, h, i\},\{i, k, l\},\{m, n, o\},\{p, q, r\}\}
$$

This is the reading in which each jury and committee gathers by itself.

The derivation (3).(2),(3),(4) yields the second reading, in which the verb is partially distributed over the outer level. The derivation produces a range of quantification whose extension is:

$$
\{\{a, b, c, d, e, f, g, h, i\},\{j, k, I, m, n, o, p, q, r]\}
$$

This is the reading in which the jurors gather in one place and the committee members in another.

Finally, the derivation (2),(3),(3),(4) yields the third reading, which is completely collective. This derivation produces a range of quantification whose extension is:

$$
\{\{a, b, c, d, e, f, g, h, i, j, k, l, m, n, 0, p, q, r\}\}
$$

This is the reading in which all persons who are either jurors or committee members gather.

\section{OTHER PARTS OF SPEECH}

In this section we discuss distributional considerations for argument-taking parts of speech other than vers - specifically prepositions and adjectives. Prepositions in our system are translated as two-place predicates, adjectives as one-place predicates. The distributional issues they raise are therefore treatable by the same machinery we have developed for transitive and intransitive verbs.

\subsection{Prepositions}

Prepositions are subject to distributional considerations that are analogous to those of transitive verbs. Consider:

The books are on the shelves

Given facts about physical objects and spatial location, the most plausible meaning for this sentence is that every book is on some shelf or other. This would be expressed by the transtation rule:

$\lambda u, v: \forall x \in \operatorname{PARTS}(u): \exists y \in \operatorname{PARTS}(v):$ ON' $(x, y)$

Note the similarity with the translation rule for "CONTAIN". from which it differs in that the roles of the first and the second argument in the quantificational structure are reversed.

\subsection{Adjectives}

The treatment of adjectives in regular form is exactly analogous with that given intransitive verbs such as "walk". Thus, for the adjective "red", we may have the translation rule:

$$
\text { RED } \Rightarrow \lambda u: \forall x \in \operatorname{PARTS}(u): \operatorname{RED}(x)
$$

A more interesting problem is seen in sentences containing the comparative form of an adjective, as in:

The trigates are faster than the carriers

What are the truth-conditions of this sentence? One might construe it to mean that every frigate is faster than every carrier, but this seems unneccesarily strong. Intuitively, it seems to mean something a little weaker than that, allowing perhaps for a few exceptions in which a particular carrier is not faster than a particular frigate.

On the other hand, another requirement eliminates truth-conditions which are too weak. For if "The frigates are faster than the carriers" is true, it must surely be the case that "The carriers are faster than the frigates" is false. This requirement holds not only for "faster", but for the comparative form of any adjective.

The treatment of comparative forms in the Spoken Language System can be illustrated by the following schema:

$$
(\lambda x, y \text { : larger }(<u t>(x),<u f>(y)))
$$

in which '<uf>' is filled in by an "underlying tunction" particular to the adjective in question. For the adjective "fast", this underlying function is "speed".

The requirement of anti-symmetry for the distributions of comparatives is now reduced to a requirement of anti-symmetry for the distributional translation of the EFL constant "larger". In this way, the antisymmetry requirement is expressed for all comparatives at once.

Obviously anti-symmetry is fufilled for the universal-universal translation, but, as we have pointed out, this is a very strong condition. There is another, weaker condition which fufills anti-symmetry:

$$
\begin{aligned}
& \text { larger' } \Rightarrow \\
& \lambda u, v: \forall x \in \text { PARTS }[u]: \exists y \in \text { PARTS }[v] \text { : larger' }[x, y] \wedge \\
& \forall x \in \text { PARTS }[v]: \exists y \in \text { PARTS }[u]: \text { larger' }[y, x]
\end{aligned}
$$

When applied to the sentence above, this condition simply states that for every frigate there exists a carrier that is slower than it. and comversely, for every carrier there exists a frigate that is faster than it.

This is anti-symmetric as required. For if there is some frigate that is faster than every carrier, there cannot be some carrier that is faster than every frigate.

\section{THE ALGORITHM}

The algorithm which applies this method is an extension of the previously-mentioned procedure of generating all possible WML expansions from an EFL expression and weeding out semantically anomalous ones. The two steps of generate and test are now embedded in a loop that simply iterates until all EFLlevel constants, including 'PARTS', are expanded 
away. This gives us a breadth-first search of the possible recursive expansions of 'PARTS', one which nevertheless does not fail to halt because semantically anomalous versions, such as those attempting to quantify over expressions which are not sets, or those applying descriptive relations to arguments of the wrong type, are weeded out and are not pursued any further in the next iteration.

We can now define the function TO-WML, which takes an EFL expression and produces a set of WML expressions without EFL constants. $I$ is:

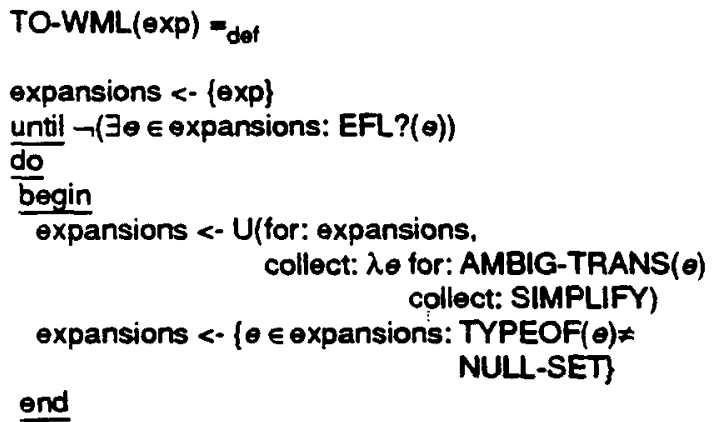

The function AMBIG-TRANS expands the EFL-level constants in its input, and returns a set of expressions. The function EFL? returns true if any EFL constants are present in its argument. The function TYPEOF takes an expression and returns its type; it retums the symbol NULL-SET if the expression is semantically anomalous. Note that if a particular expansion is found to be semantically anomalous it is removed from consideration. If no non-anomalous expansion can be found the procedure halts and the empty set of expansions 0 is returned. In this case the entire EFL expression is viewed as anomalous and the interpretation which gave rise to it can be rejected.

\section{CONCLUSIONS}

We have shown how treatments of the collective/distributive distinction must take into account the phenomenon of "partial distributivity", in which a collective verb optionally distributes over the outer levels of structure in what we call a "multi-level" plural. Multiple levels of structure must be allowed in the semantics of such plural NPs as "the boys and the giris", "the committees", etc.

We have presented a computational mechanism which accounts for these phenomena through a framework of recursive translation rules. This ramework generates quantifications over alternative levels of plural structure in an NP, and can handle NPs of arbitrarily complex plural structure. It is economical in its means of producing arbitrary numbers of readings: the multiple readings of the sentence such as The juries and the committees gathered" are expressed with just one translation rule.

\section{References}

[1] Bartsch, $\mathbf{R}$

The Semantics and Syntax of Number and Numbers.

In Kimball. J.P. (editor), Syntax and Semantics, Vol. 2. Seminar Press, New York, 1973.

[2] Bennett, M.R

Some Extensions of a Montague Fragment of English.

Indiana University Linguistics Club. 1975.

[3] W.J.H.J. Bronnenberg, H.C. Bunt, S.P.J. Landsbergen, R.J.H. Scha, W.J. Schoenmakers and E.P.C. van Utteren.

The Question Answering System PHLIQA1.

In L. Bolc (editor). Natural Language Question Answering Systems. Macmillan, 1980.

[4] Landman, Fred.

Groups.

1987.

University of Massachusetts, Amherst.

[5] Landsbergen, S.P.J. and Scha, R.J.H.

Formal Languages for Semantic Representation.

In Allén and Petöfi (editors), Aspects of Automatized Text Processing: Papers in Textlinguistics. Hamburg: Buske, 1979.

[6] Montague, R.

The Proper Treatment of Quantification in Ordinary English.

In J. Hintakka, J.Moravcsik and P.Suppes (editors), Approaches to Natural Language. Proceedings of the 1970 Stanford Workship on Grammar and Semantics. pages 221-242. Dordrecht: D.Reidel, 1973.

[7] Scha, Remko J.H

Distributive, Collective and Cumulative Quantification.

In Jeroen Groenendijk, Theo M.V. Janssen. Martin Stokhof (editors). Formal Mothods in the Study of Language. Part 2, pages 483-512. Mathematisch Centrum, Amsterdam, 1981.

[8] Scha, Remko J.H.

Logical Foundations for Question-Answering.

Philips Research Laboratories, Eindhoven, The Netherlands, 1983.

M.S.12.331.

[9] Stallard, David G.

The Logical Analysis of Lexical Ambiguity. In Proceedings of the ACL. Association for Computational Linguistics, July, 1987. 